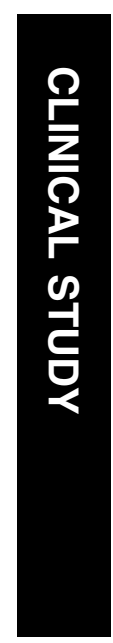

\title{
A population-based study of Coats disease in the United Kingdom II: investigation, treatment, and outcomes
}

\begin{abstract}
Aims Coats disease is an uncommon form of retinal telangiectasia. We conducted a prospective population-based study of Coats disease in the United Kingdom to provide a more balanced picture. This paper reports the investigations and treatments used for Coats disease and their anatomic and visual outcomes.
\end{abstract}

Methods The study was conducted through the British Ophthalmic Surveillance Unit. All ophthalmologists notifying cases of Coats disease were sent a baseline questionnaire and a follow-up questionnaire after 6 months.

Results In total, 55 baseline and 42 follow-up questionnaires were returned. All cases were unilateral. Ultrasound was performed in $26 \%$ of cases, fluorescein angiography in $35 \%$, and examination under anaesthesia in $42 \%$ of the cases. Laser photocoagulation was by far the primary treatment modality, used in $92 \%$, with cryotherapy used mainly as a second-line or adjunctive treatment. In more advanced cases with significant retinal detachment, laser photocoagulation combined with pars plana vitrectomy and drainage of sub-retinal fluid can produce anatomic stability and prevent progress to end-stage disease. Intravitreal injections of steroids or VEGF inhibitors are currently of unproven efficacy in Coats disease. Overall, treatment resulted in stabilisation of visual acuity. Anatomic stabilisation or improvement was achieved in virtually all eyes.

Conclusion Laser photocoagulation to telangiectatic retinal vessels is the most commonly used treatment for Coats disease. Other treatments are either adjunctive or of unproven efficacy. A realistic treatment goal is to achieve anatomic stability and avoidance of enucleation for painful end-stage disease. Visual improvement is however unlikely. Eye (2010) 24, 1802-1807; doi:10.1038/eye.2010.127; published online 24 September 2010

Keywords: Coats disease; retinal exudation; laser photocoagulation

\section{Introduction}

Coats disease is a well-described but uncommon form of retinal telangiectasia characterised by intra-retinal and sub-retinal exudation in the absence of significant vitreoretinal traction. ${ }^{1}$ More advanced cases may progress to complete retinal detachment, neovascular glaucoma, and sometimes require enucleation. ${ }^{1-3}$ Although there are published case series of Coats disease, no populationbased studies have been performed. We have conducted a prospective population-based study of Coats disease in the United Kingdom to provide a balanced picture of the disease spectrum. An accompanying paper describes the basic demographic data and clinical features at diagnosis. This paper reports the investigations used in the assessment of cases of Coats disease. We also report the treatments used and their anatomic and visual outcomes 6 months after presentation. This information will hopefully be very informative to clinicians and should allow them to plan treatment for patients with Coats and to counsel patients and carers of children with this condition. 


\section{Materials and methods}

This was a prospective population-based study of Coats disease in the United Kingdom conducted through the British Ophthalmic Surveillance Unit (BOSU). All consultant ophthalmologists in the United Kingdom are sent a monthly reporting card by BOSU, which at any time enquires about several ophthalmic conditions. BOSU in turn inform the investigators who then send a questionnaire to the reporting ophthalmologist. The design and clinical relevance of all projects using BOSU are assessed by their scientific advisors and all require full ethical assessment and approval. All

ophthalmologists reporting cases of Coats disease to the investigators were sent a baseline questionnaire and a follow-up questionnaire 6 months later. Ethical approval for this study was obtained from Fife, Forth Valley, and Tayside Research Ethics Service, REC reference 06/S0501/43. The ethical approval permitted the investigators to send reminders for unreturned questionnaires though no other contact was permitted.

The Coats disease study was on the BOSU reporting cards for 12 months from January 2008, the 100th anniversary of George Coats original publication. ${ }^{4}$ The baseline questionnaire enquired about age at presentation, gender, mode of presentation, initial LogMAR visual acuity, and clinical features. The clinical features included anterior and posterior segment findings, number of clock hours of retinal exudation, and disease staging. The staging of Coats disease followed that described by Shields. ${ }^{5}$

We enquired about investigations carried out and the treatments used. Patients with associated ophthalmic disorders, such as retinitis pigmentosa and underlying medical conditions and syndromes, were excluded. The follow-up questionnaire at 6 months later enquired about LogMAR visual acuity, clinical features, disease stage, and whether any additional investigations or treatments were required. Given the volume of information, we have reported the data in two separate papers. This paper reports the investigations and treatments performed and their outcomes. An accompanying paper reports on the epidemiology and clinical features at presentation.

\section{Results and conclusion}

A total of 55 completed baseline questionnaires for eligible cases of Coats disease were returned to the authors. We received completed 6-month follow-up questionnaires for 42 cases $(76.4 \%)$. This paper reports on the preferences of the reporting ophthalmologists for investigation and treatment of Coats disease. We also report changes in visual acuity as well as structural outcomes (Coats disease staging). A 6-month follow-up data were available for 41 eyes as 42 of the 6-month follow-up questionnaires were returned with one case not eligible for inclusion because of early enucleation.

\section{Investigations}

A B-scan ultrasound was performed in 14 cases (25.5\%), fundus fluorescein angiography (FFA) in 19 cases $(34.5 \%)$, and examination under anaesthesia in 23 cases $(41.8 \%)$. The mean age of patients undergoing FFA was 168 months with 11 under 120 months of age. This suggests that many departments now possess a portable FFA system that can be used in children under general anaesthesia. Computerised tomography scanning was carried out in just two children, aged 11 months and 61 months, respectively, presumably to out-rule retinoblastoma. Only the 11-month-old child however had leucocoria as the presenting feature. Two investigations were performed in $27.2 \%$ and three investigations in $7.3 \%$ of cases. In 18 cases (32.7\%), no investigations were carried out in addition to the clinical examination.

\section{Treatment or observation}

A decision to treat was taken in 52 out of 55 cases of Coats disease $(94.5 \%)$. The remaining three cases $(5.5 \%)$ were observed. Enucleation was carried out in just one case $(1.8 \%)$ due partly to concern about a possible retinoblastoma in an 11-month-old child and partly to a very advanced disease with rubeotic glaucoma (stage 4 ).

Of 52, 48 treated eyes received various forms of laser photocoagulation, Figure 1. Argon laser

photocoagulation was the most commonly used with 32 eyes treated. Diode laser was used in five cases, frequency-doubled YAG (FD-YAG) laser in three cases. In eight cases the type of laser used was not specified. Retinal cryotherapy was used in 10 cases; in 4 cases

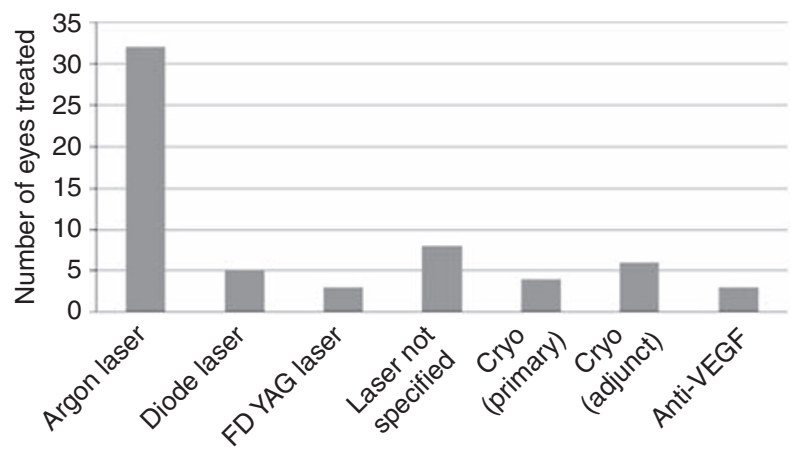

Figure 1 Initial treatments used in Coats disease. 
Table 1 Primary treatment of Coats: percentage of cases with each disease stage receiving different treatments

\begin{tabular}{|c|c|c|c|c|c|c|}
\hline Disease stage & Diode laser & Argon laser & FD-YAG laser & Cryotherapy & Argon + cryo & $P P V$ \\
\hline $2 \mathrm{~A}(n=8)$ & 38 & 38 & 0 & 12 & 0 & 12 \\
\hline $2 \mathrm{~B}(n=18)$ & 11 & 61 & 11 & 6 & 11 & 0 \\
\hline $3 \mathrm{~A}(n=13)$ & 0 & 62 & 0 & 8 & 15 & 15 \\
\hline $3 \mathrm{~B}(n=5)$ & 0 & 40 & 0 & 0 & 40 & 20 \\
\hline
\end{tabular}

Abbreviations: Cryo, cryotherapy; FD-YAG, frequency-doubled YAG laser; PPV, pars plana vitrectomy.

cryotherapy was the primary treatment, whereas in 6 cases it was used as an adjunct to laser. Many younger patients received argon laser photocoagulation, with 19 of them being aged 120 months or less. This suggests that many departments now possess portable argon laser systems and most are not reliant on diode lasers as they were in the past. The choice of primary treatment modality varied with the disease stage at presentation (Table 1). In stage 2A, $76 \%$ of eyes received laser (argon or diode), whereas $12 \%$ (one eye) received cryotherapy and $12 \%$ pars plana vitrectomy with laser. In stage $3 \mathrm{~B}$, $40 \%$ received laser alone, $40 \%$ laser and cryo, and $20 \%$ pars plana vitrectomy (Table 1).

An additional question asked was, whether laser treatment was applied directly to the telangiectatic vessels and or as a barrier around the area of retinal exudation. In total, 29 respondents answered this question. In all cases laser photocoagulation was applied to the telangiectatic vessels and in nine cases it was additionally applied as a barrier.

Intravitreal injections of vascular endothelial growth factor (VEGF) inhibitors were only used as adjunctive therapy and with no marked difference in outcomes, though the numbers are small (seven injections of bavacizumab to four eyes). In addition, the eyes that received VEGF injections had on an average more advanced disease at presentation; stage 2B (two eyes), 3A and $3 \mathrm{~B}$ (one each). No ocular or systemic side effects were reported after the use of VEGF inhibitors, though we did not specifically enquire for these.

\section{Additional treatment}

Among the 41 eyes in which follow-up data were available, $23(56 \%)$ received additional treatment. In total, 21 were treated with laser (argon $n=12$, FD-YAG $n=6$, and diode $n=3$ ). Six of the eyes that received additional laser treatment also received adjunctive cryotherapy $(n=3)$ or intravitreal bevacizumab $(n=3)$. Two eyes were treated with cryotherapy alone as their second treatment. Finally, one eye with stage 4 Coats disease and glaucoma required topical agents to lower intraocular pressure.
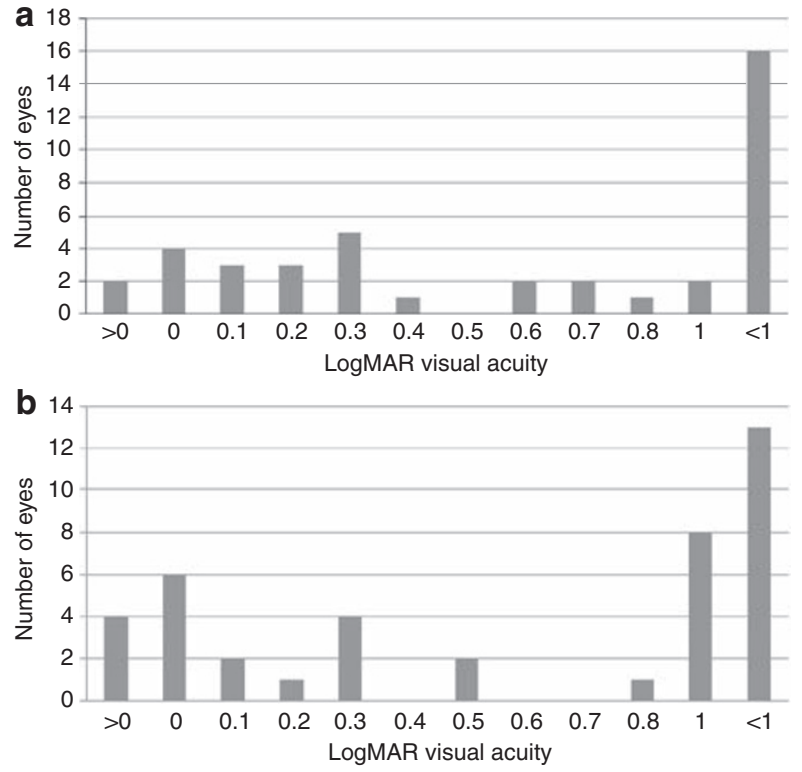

Figure 2 (a) Visual acuity at diagnosis. (b) Visual acuity at 6-month follow-up.

\section{Visual acuity outcome}

Figures 2a and b show baseline and 6-month follow-up visual acuity results respectively. For clarity, we have included the baseline visual acuity only for the 41 cases in which a 6-month follow-up data were returned. Vision improved in 13 eyes (32\%), worsened in 11 eyes (27\%), and remained stable in 17 eyes $(41 \%)$. The number of eyes with LogMAR vision of 1.0 or worse increased slightly from 18 to 21 (51\%). However, the number of eyes with LogMAR vision of 0 or better increased from 6 to $10(24 \%)$. Among the 13 eyes in which vision improved, all had received treatment. Twelve of these eyes had laser and two 2 had cryopexy and intravitreal bevacizumab (one eye had cryopexy as primary and one as adjunctive treatment). Eleven eyes (27\%) had worse vision at follow-up and all had been treated, including one eye that had drainage of sub-retinal fluid and intravitreal bevacizumab. In the three cases that did not receive any treatment, vision was unchanged at the 6-month follow-up. We looked at age at presentation as a predictor for visual improvement. The percentage of eyes 
with improved vision at 6-month follow-up was 33\% in those aged less than 5 years; $13 \%$ for those between 5 and 10 years of age; $10 \%$ between 10 and 15 years; and $67 \%$ more than 15 years of age.

\section{Anatomic outcomes}

The Coats staging worsened in only one case, but vision remained unchanged ( $\log M A R=0)$, treatment with diode laser was administered to this eye. There was an improvement in the staging of 16 eyes (39\%) whereas 24 eyes $(59 \%)$ were unchanged. Among the eyes with improved staging, all were treated. Eleven of the treated eyes received laser alone; three eyes received laser and cryotherapy, and two received cryotherapy alone. In addition, two of the treated eyes received intravitreal injections (steroid $n=1$; bevacizumab $n=1$ ). In all three eyes that were observed rather than treated, staging was unchanged at the 6-month follow-up. Figures $3 a$ and $b$ show the number of eyes in the various Coats stages at baseline-diagnosis and again at 6-month follow-up. Again, we have included the baseline staging only for the eyes in which 6-month follow-up data were returned. The most notable feature is the marked improvement in staging of many eyes. At baseline, no eyes were classified as stage 1 , whereas at 6 months, nine eyes (22\%) were classified as stage 1 . The proportion of eyes with stages 2 or 3 at presentation that had an improvement in their Coats stage after treatment (at 6 month follow-up) was approximately equal to 31 and $29 \%$, respectively.
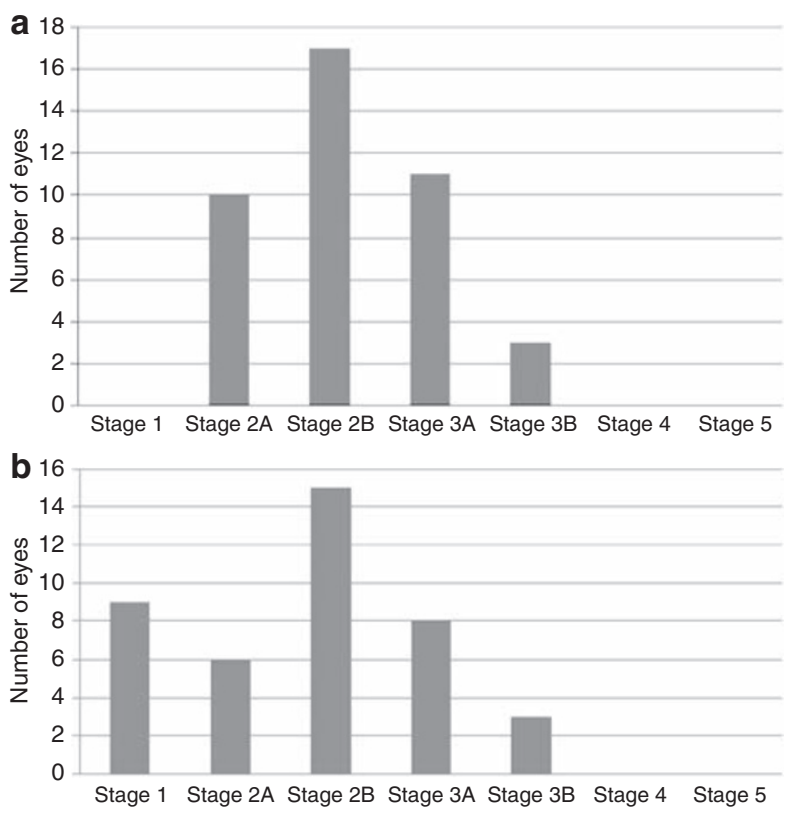

Figure 3 (a) Coats stage at diagnosis. (b) Coats disease stage at 6-month follow-up.
There was no significant additional benefit of either visual or structural outcome in eyes that had a laser barrier.

\section{Discussion}

This study is the first ever prospective population-based study of Coats disease. The accompanying paper reports the estimated incidence of Coats disease and characterises the clinical features at presentation. This paper reports the investigations performed, treatment preferences, and their visual and anatomic outcomes.

Coats disease occurs predominantly in young children and frequently presents with leucocoria, therefore other conditions must be out-ruled, especially retinoblastoma. ${ }^{6-8}$ It is not surprising therefore that an examination under anaesthesia was performed in a significant proportion of cases $(42 \%)$, and that ultrasound (26\%) and FFA (35\%) were also used extensively.

Somewhat surprisingly in our series, in 18 cases $(32.7 \%)$ no investigations were carried out in addition to the clinical examination. Shields et $a l^{1}$ reported a series of 150 patients (158 eyes) in which ultrasound was used in $48 \%$ of eyes and fluorescein angiography in $31 \%$.

Telangiectasia in Coats disease show early leakage into adjacent retina. ${ }^{1}$ Ultrasound demonstrates distinctive medium reflective echoes from the sub-retinal space and is particularly helpful in excluding retinoblastoma. ${ }^{1,7}$ Optical coherence tomography was neither included in our questionnaires nor mentioned by any respondents, it could however be useful as a non-invasive means of evaluating children with leucocoria. ${ }^{9}$

We have demonstrated modest overall visual improvements in our series of Coats disease with the number of eyes with improved vision slightly more than the number with worsened vision. Our study was however limited to 6-months follow-up and the final visual outcomes could differ. Stabilisation of visual acuity and perhaps improvement in some eyes is therefore a realistic treatment goal. The proportion of eyes with very poor vision ( $\log M A R$ vision $\leq 1)$ was $51 \%$ in those eyes for whom we have 6-month follow-up data. This figure compares well with existing published series and is most likely because of the population-based nature of our study. Two series from large tertiary referral centres have significantly worse proportions of eyes with very poor vision; 64\% for Shields (Wills Eye Hospital, Philadelphia, PA, USA) ${ }^{5}$ and $96 \%$ for Budning (Hospital for Sick Children, Toronto, ON, Canada). ${ }^{3}$

We have shown that it is possible to achieve stabilisation or improvement of the Coats staging in virtually nearly all cases (98\%). The disease stage at diagnosis is therefore a highly important prognostic factor in this series. In Shields' series in which the same 
classification system was used, the Coats stage was stable or improved in $76 \%$ of eyes with the best prognosis for eyes with milder disease-stage at diagnosis. ${ }^{5}$ Shields' series had a mean follow-up of 55 months (range, 6 months to 25 years) and found recurrence of retinal telangiectasia and exudation in $7 \%$ of cases by 10 years. ${ }^{5}$ Other reports have also confirmed that prognosis was related to the number of clock hours of retinal exudation present at diagnosis and not to the patients age. ${ }^{3,10,11}$ Smithen et $\mathrm{l}^{11}$ reported a series of Coats disease in adults, most of whom had extensive retinal exudation and in whom vision worsened in $62 \%$ despite treatment. The extent or stage of Coats disease at diagnosis would therefore seem to be the single most important prognostic factor.

The aim of treatment in Coats disease is the eradication of telangiectasia leading to resolution of retinal exudation with the ultimate goal of salvaging as much vision as possible. ${ }^{5}$ In Shields large series, cryotherapy was the most commonly used therapy followed by laser photocoagulation and with no serious treatment complications. ${ }^{5}$ Sheilds' series was however retrospective and over two decades, during that period there was a shift away from cryotherapy towards an increased use of laser in the treatment of Coats disease..$^{5}$ In our study, laser photocoagulation was by far the most popular treatment modality used for Coats disease, accounting for $92 \%$ of primary treatments. Cryotherapy was used mainly as a second-line or adjunctive treatment. Laser photocoagulation, repeated where necessary, is now the first-line treatment in all recent reports on Coats disease. ${ }^{2,3,10-12}$ In more advanced cases with significant retinal detachment and poor prognosis, laser photocoagulation perhaps combined with pars plana vitrectomy and drainage of sub-retinal fluid can stabilise the disease and prevent progress to end-stage disease. $9,12,13$ As discussed above, a realistic treatment goal in eyes with retinal detachment is anatomic improvement, without an increase in visual function, and avoidance of enucleation for painful end-stage disease. ${ }^{13}$

Elevated intraocular levels of VEGF have been documented in an advanced case of Coats disease in a 2-year-old boy with retinal detachment refractive to surgical treatment. ${ }^{14}$ This child received two intraocular injections of pegaptanib, which produced both anatomic improvement and a marked reduction in intraocular VEGF levels. ${ }^{14}$ Another case report however found an equivocal benefit from intravitreal injections of triamcinolone and bevacizumab. ${ }^{15}$ Intravitreal injections of VEGF inhibitors were only used as an adjunctive therapy in a small number of eyes in our series. The VEGF inhibiting agents had no marked effect on outcome, though treated eyes had on average more advanced disease. Bergstrom et al ${ }^{16}$ reported five children (ages 3-12 years) with unilateral Coats disease and retinal detachment that were treated with cryotherapy and intravitreal triamcinolone injections. In Bergstrom's series of five eyes, four developed glaucoma, three severe cataract and three eyes developed inoperable retinal detachments. ${ }^{16}$ Although these complications have been reported in adults they are not as common or severe, ${ }^{17}$ it may therefore be best to avoid the use of intravitreal steroid injections in Coats disease.

We have described the investigations, treatments, and outcomes in a prospective, population -based study of Coats disease. Although visual acuity improvement is unlikely in most cases, there was a much more encouraging prospect for anatomic improvement. The conditions of the ethical approval for this study limited us to just one follow-up questionnaire at 6 months after diagnosis. It is possible therefore that longer follow-up might reveal further improvements in either vision and or staging as the resolution of retinal exudation is a slow process. Nonetheless, the improvements in staging are themselves very encouraging as left untreated, many cases of Coats disease progress to complete retinal detachment with painful rubeotic glaucoma.

\section{Summary}

What was known before

- Outcomes in Coats disease are frequently poor and many eyes require enucleation.

- Variety of treatment modalities described in literature.

- Some case reports/series of intravitreal injection therapies.

What this study adds

- Although vision usually does not improve, structural improvement is possible and virtually all eyes can be saved.

- Laser photocoagulation is by far the most popular primary treatment used in the UK.

- Intravitreal injection therapies are of unproved efficacy.

\section{Conflict of interest}

The authors declare no conflict of interest.

\section{Acknowledgements}

We thank Mr Barny Foot of the British Ophthalmic Surveillance Unit for his help and encouragement with this project. This project received funding from the following charitable/non-commercial sources that exercised no influence over the design or the study or analysis of data: the Eyecare fund, Princess Alexandra Eye Pavilion, Edinburgh and Sick Kids Friends Foundation, Royal Hospital for Sick Children, Edinburgh. 


\section{References}

1 Shields JA, Shields CL, Honavar SG, Demirci H. Clinical variations and complications of Coats disease in 150 cases: The 2000 Sanford Gifford Memorial Lecture. Am J Ophthalmol 2001; 131(5): 561-571.

2 Cahill M, O'Keefe M, Acheson R, Mulvihill A, Wallace D, Mooney D. Classification of the spectrum of Coats' disease as subtypes of idiopathic retinal telangiectasis with exudation. Acta Ophthalmol Scand 2001; 79: 596-602.

3 Budning AS, Heon E, Gallie BL. Visual prognosis of Coats disease. J Am Assoc Pediatr Ophthalmol Strabismus 1998; 2: 356-359.

4 Coats G. Forms of retinal diseases with massive exudation. Roy London Ophthalmol Hosp Rep 1908; 17: 440-525.

5 Shields JA, Shields CL, Honavar SG, Demirci H, Cater J. Classification and management of Coats disease: the 2000 Proctor Lecture. Am J Ophthalmol 2001; 131(5): 572-583.

6 Shields JA, Shields CL. Coats disease: the $2001 \mathrm{LuEsther} \mathrm{T}$ Mertz lecture. Retina 2002; 22(1): 80-91.

7 Shields JA, Shields CL. Differentiation of Coats disease and retinoblastoma. J Am Assoc Pediatr Ophthalmol Strabismus 2001; 38: 262-266.

8 Char DH. Coats' disease: long term follow up. Br J Ophthalmol 2000; 84(1): 37-39.

9 Shields CL, Mashayekhi A, Luo CK, Materin JA, Shields JA. Optical coherence tomography in children: analysis of 44 eyes with intraocular tumors and simulating conditions. J Pediatr Ophthalmol Strabismus 2004; 41(6): 338-344.

10 Adam RS, Kertes PJ, Lam WC. Observations on the management of Coats disease: less is more. Br J Ophthalmol 2007; 91(3): 303-306.

11 Smithen LM, Brown GC, Brucker AJ, Yannuzzi LA, Klais CM, Spaide RF. Coats disease in adulthood. Ophthalmol 2005; 112(6): 1072-1078.

12 Schefler AC, Berrocal AM, Murray TG. Advanced Coats disease: management with repetitive aggressive laser ablation therapy. Retina 2008; 28(3 suppl): S38-S41.

13 Mrejen S, Metge F, Denion E, Dureau P, Edelson C, Caputo G. Management of retinal detachment in Coats disease. Retina 2008; 28(3 suppl): S26-S32.

14 Jain SY, Moshfeghi DM. Elevated vascular endothelial growth factor levels in Coats disease: rapid response to pegaptanib sodium. Graefes Arch Clin Exp Ophthalmol 2007; 245(9): 1387-1388.

15 Jun JH, Kim YC, Kim KS. Resolution of severe macular oedema in adults Coats disease with intravitreal triamcinolone and bevacizumab injection. Korean J Ophthalmol 2008; 22(3): 190-193.

16 Bergstrom CS, Hubbard GB. Combination intravitreal triamcinolone injection and cryotherapy for exudative retinal detachments in severe Coats disease. Retina 2008; 28(3 suppl): S33-S37.

17 Thompson JT. Cataract formation and other complications of intravitreal triamcinolone for macular edema. Am J Ophthalmol 2006; 141(4): 629-637. 